\title{
Effects of Strength Training on Physical Fitness and Obesity of College Girls of Yazman,
}

\author{
Bahawalpur, Pakistan \\ * Adeela Rauf, MPhil Scholar \\ ** Sumbal Kaynat, Lecturer
}

*** Muhammad Zia ul Haq, Assistant Professor (Corresponding Author)

\begin{abstract}

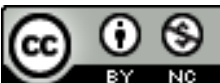

The purpose of this study was to examine the influence of strength training on physical fitness and obesity of college girls. The selected $(n=70)$ participants were girls' students of Yazman, Bahawalpur. The selected variables were $600 \mathrm{~m}$ race for endurance, $30 \mathrm{~m}$ dash for speed, standing broad jump for leg power, zigzag run for agility, sit and reach for flexibility, and handgrip streng th. The body composition was estimated through five skin folds, seven girths, three breadths, and the lengths of upper and lower limbs. Eight weeks training schedule was adopted, (five days in a week) which comprises of different physical activities such as a $400 \mathrm{~m}$ race, sit-ups, skipping, matches of badminton and volleyball. A paired t-test was applied to examine the effect of training on body composition and physical fitness of college girls. The result showed that strength training significantly affects bicep skin fold, hip girth, chest girth, 30m race, and flexibility. It is concluded that strength training increases physical fitness and reduces the body fat of college girls. The comparative analysis results revealed that after eight-week strength training, the college girls' students become physically active, reduced body fats, and assist in weight management.
\end{abstract}

Keywords: Physical Fitness, Body Composition, Girls' Students, Strength Training

\section{Introduction}

The physical fitness of college students reduces cardiovascular diseases, heart strokes, diabetes, and all mortality causative issues (Fu, Goa, Hannon, \& Shul, 2013). From the last few decades, obesity and overweight considerably increased in teen ages thorough out the world and more than 30 percent population is characterized as obese across the globe (Calestine, Boop, \& Papalia, 2017). Obesity has become one of the most well-known medical conditions in students which are characterized as extra body fat and negatively affect health (Egli, Bland, \& Melton, 2011). Several studies have highlighted that obesity is correlated with a reduction in physical activities and extreme intake of nutrition (Zheng, Lan, \& Yan, 2013). Although health care efforts aimed to encourage teenage students to eat healthy food and do more exercise, not a single country has yet been succeeded in reversing the prevalence of obesity in the last many years (Zhao, Wei, \& Li, 2011).

Exercise and physical training regarding guidelines by public health also added marvelous contributions to the physical fitness of the students and provided direction on the intensity, frequency, and duration of physical activities (Lockwood \& Wohl., 2012). For instance, the US Centres for Disease Control and Prevention suggests at least 30 minutes and preferable 60 minutes exercise in at least 5 days per week to adults of 18 to 64 years can provide health benefits (French \& Jeffery., 1994). Similar recommendations have been published by the World Health Organization (WHO) and aimed to decrease $10 \%$ occurrence is insuffic ient physical activities (Weinstock, Capizzi, Weber, \& Petry, 2014). When students shift from high schools to colleges, they gain more autonomy as compared to their everyday lives (Farren, Zhang, \& Thomas, 2017). Epidemiological findings have testified a significant reduction in physical activities because of increased independence throughout high school and college level (Gutin, Cucuzzo, \& Stachura., 1996). Ordinary-weight students had $11 \%$ higher grades on average as compared to overweight students (Stice, Shaw, \& Marti, 2006). There is very

* Department of Physical Education and Sports Science, The Is lamia University of Bahawalpur, Pakistan Email: adeelarauf.30@gmail.com

** Capital University of Physical Education \& Sports, Beijing, China/Department of Physical Education, NUMAL University Islamabad, Pakistan Email: sumbal.kaynat@numl.edu.pk

*** Department of Physical Education and Sports Science, The Islamia University of Bahawalpur, Pakistan Email: muhammad.zia@iub.edu.pk 
limited research examining how physical fitness, activity, and academic results are correlated throughout the college years (Puhl \& Heuer, 2009). Physical inactiveness and obesity among college girls have imposed a major public health concern (Sanchez, Rosas, Beak, \& Egerter, 2012). Components of physical fitness include body composition, muscle endurance, muscle strength, cardiopulmonary functions, agility, and flexibility (Lauderdale, Irwin, \& Layne, 2015). Regular physical activities reduce the percentage of body weight, body fat, enhance physical health and fitness among obese girls of colleges (Sibley, Hancock, \& Bergman, 2013).

Furthermore, the link between physical fitness and body composition among college students is a lack of evidence (Downs \& Ashton., 2011). Thus, this study is filling the research gap, from the perspective of Pakistan. Therefore, a study needs to examine the bond between accurately measured physical fitness including muscular endurance, flexibility, agility, strength, and anthropometric variables including girth, breadth, skinfold, length some other factors like height, weight, arm spam, handgrip, and sitting height. These variables are the main cause of activeness in college girls. These are also affected by strength training and can be maintained by proper physical exercises. The significance of the study is that we come to know about the effect of strength training on physical fitness and body composition of college girls.

\section{MAte rial and Methods}

This study was conducted in the Govt. Degree College for Women, Tail Walla Bangla, and Yazman, Bahawalpur, Pakistan. The selected participants were $(n=70)$ female students of $16-20$ years. The fitness training program for this research was conducted with the approval of the college administration. The consent letter was obtained before the start of the study and the procedure of data collection was thoroughly brief to all participants. All variables were recorded by using specific sheets on a daily and weekly basis.

\section{Physical Fitness and Anthropome tric Measurements}

The weight of all participants was measured in kilograms with the help of a digital weight scale. The measurement of weight was obtained as, hands at sides, looking straight ahead, in relax and the result was captured. The height was measured by using a stadiometer as the participants were guided to look forward hanging arm at side in relaxing and the measurements were recorded from the apex of the head-to- ground as obtained by (Haq, Iqbal, Afzal, Ahmad, Abbas, \& Yaqoob, 2019). First, body marking was completed before measure the skinfolds, girths breadth, and lengths of body segments as proposed by (Meckel, Galily, Nemet, Eliakim \& Meekel, 2011). A skinfold caliper was used to measure the subscapular, triceps, biceps, iliac crest, abdomen, supraspinal, frontal thigh, and calf (Downs \& Ashton., 2011). A bone caliper was used to measure lengths of arm, hand, leg, and breadths of the head, shoulders, and leg (Carrell \& West, 2011). A measuring tape was used for the measurement of the girth of the upper and lower limb (Egli, Melton \& Bland, 2011). A large sliding bone caliper was used to measure the lengths of the upper and lower limb.

The zig-zag t-test was taken to examine the agility of college girls. Girls start running from the first cone toward straight, then turned left, then straight to the right side cone, return to the central cone, and finally return to the starting point. The measurements were noted in seconds. The participant was asked to sit on the floor, stretch straight his legs by touching her feet to the wooden box, push the sliding mark at the wooden box as far as possible without bending knees. The measurements were recorded in centimeters.

The performance of the standing broad jump was examined as participants stand at a marked line on the ground with feet slightly apart, flex their body, swing their arms, and finally jumps forward with explosive power. The measuring tape was used to measure the distance of jumped in meters. A 30-meter dash was measured with a standing start to examine speed. This is the description of $30 \mathrm{~m}$ sprint tests. This test aims to determine acceleration and speed. Handgrip strength is important for any sports in which the hands are used for catching, throwing, or lifting. Also, as a rule, people with strong hands tend to be strong elsewhere, so this test is often used as a general test of strength. Start with a hand up and bring it down to the side while pulling in the handle of the dynamometer, do not swing your hand (Steiber, 2016). Handgrip strength measurements were recorded in $\mathrm{kg}$.

\section{Training Procedure}

The 8 weeks fitness training schedule five days per week which comprises of different physical activities described (Weinstock et al, 2014). The first-day warm-up, five 30-meter dashes, and 3 sets of 20 sets of sit-ups and cool down. Second day 800-meter judging, 3 sets of 20 sit-ups, cool down 
and 30 mere slow paces walk. Third-day conduct of badminton and volleyball practices matches and 30 -meter dash, and 3 sets of skipping rope. On the fourth day skipping rope for 10 minutes, 3 sets of 30 sit-ups, and aerobics exercise with music. On the fifth day, 3 sets of the 30-meter dash, 10 minutes skipping rope, and 400-meter jogging.

Data Analysis

Descriptive statistics were applied for all variables as mean and standard deviation. A paired t-test was applied to examine the effect of strength training on physical fitness and body composition of college girls. The p. value was adjusted 0.5 for all measures. The SPSS version 25 . Was used for statistical Analysis (Pope, \& Harvey-Berino, 2013).

\section{Results}

The basic aim of the current study is to examine the influence of physical training and exercise on the fitness and obesity of female students at Govt. Degree College for Women, Bangla Tail Walla, Bahawalpur, and Pakistan. These paired t-test outcomes were performed on SPSS software between pre-and post-training exercise data.

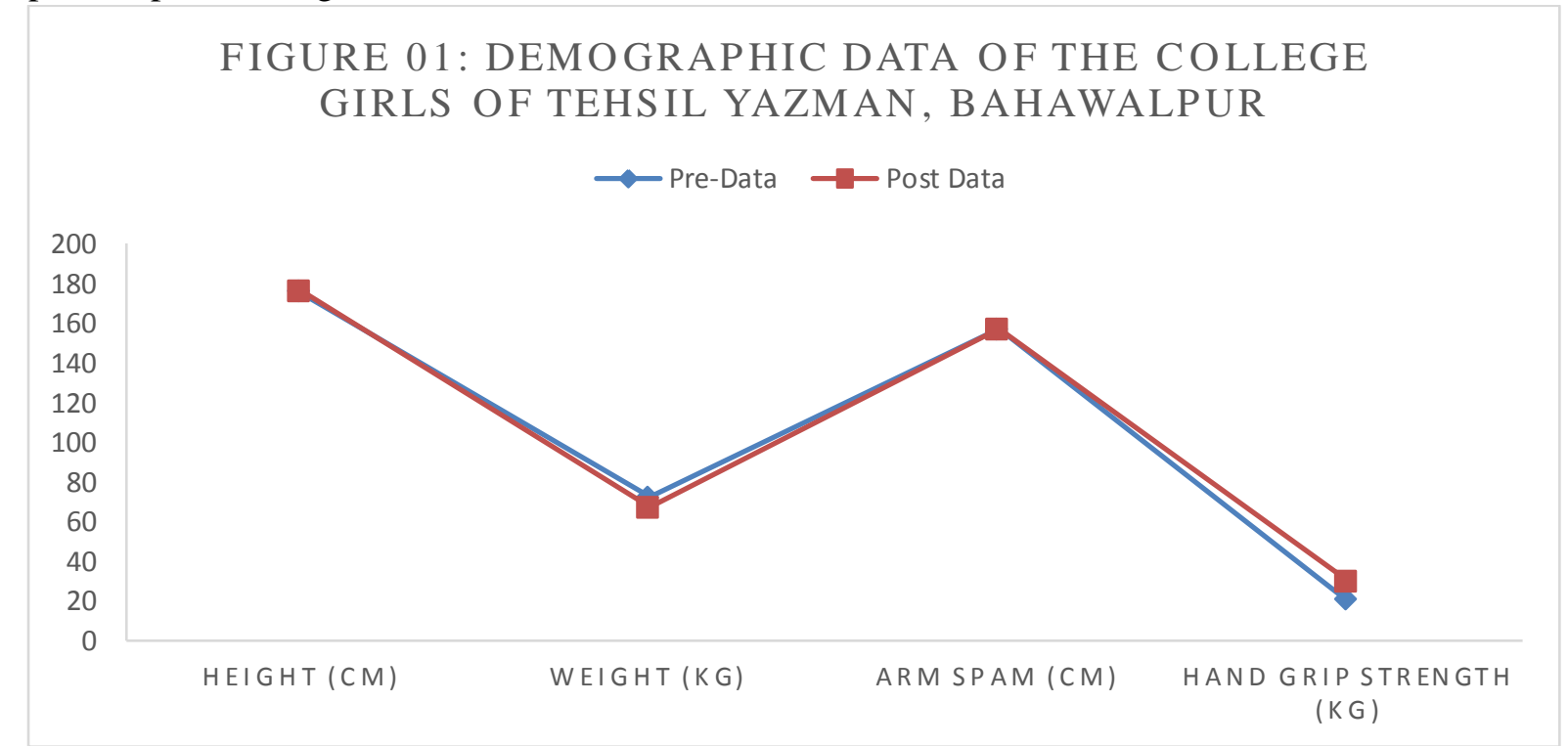

Significant at 0.5 levels.

The table shows that there was no significant difference in their arm spam, weight, and handgrip strength.

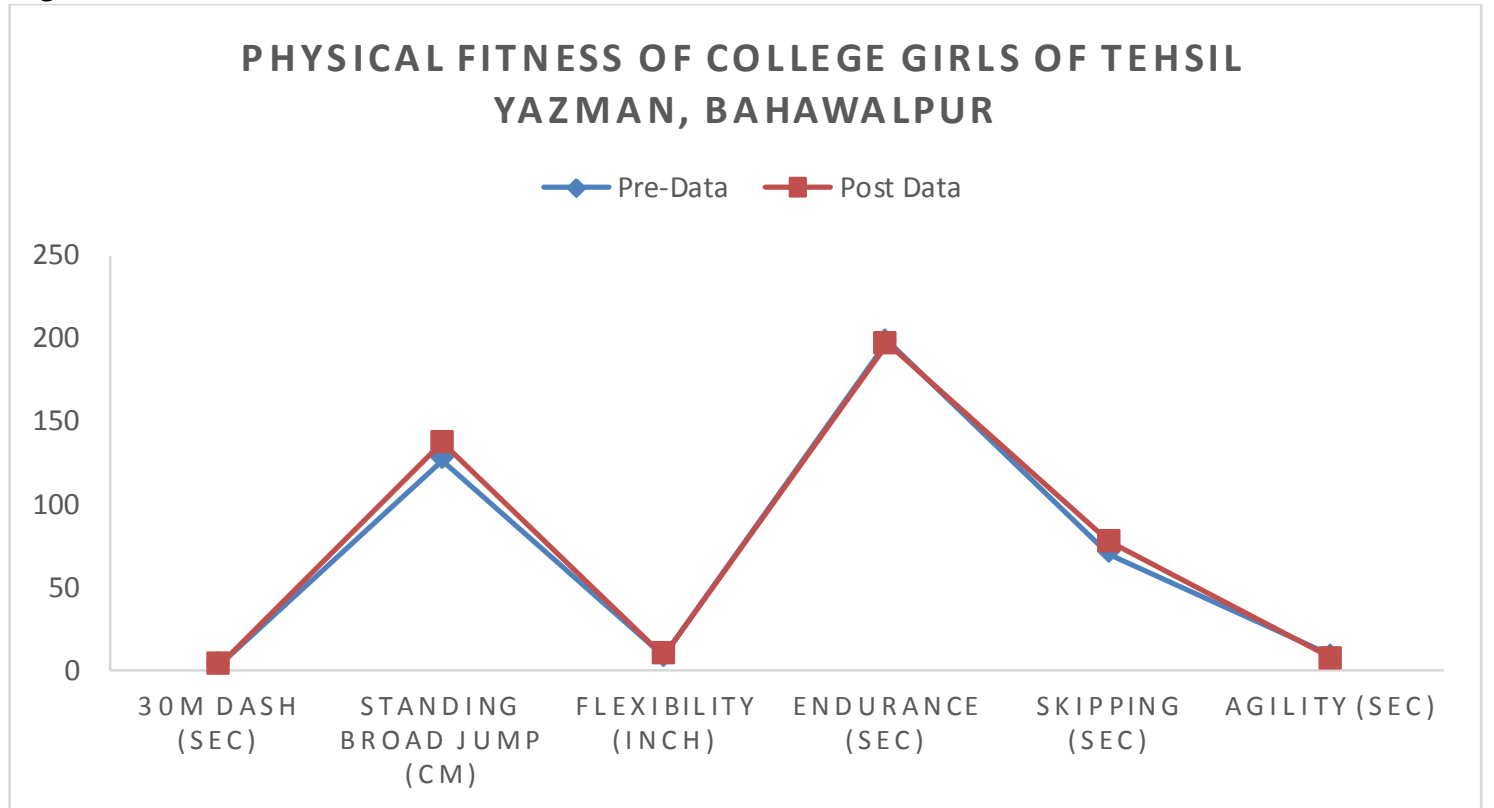

Significant at 0.5 levels.

Graph 02: Shows significant difference in pre-training and post-training leg power $t=-2.28, \mathrm{sig}=0.03$, and significant difference flexibility as pre-training and post-training $t=1.02$, sig $=0.02$. 


\section{FIGURE 03: ANTHROPOMETRIC MEASURES OF COLLEGE GIRLS OF TEHSIL YAZMAN, BAHAWALPUR}

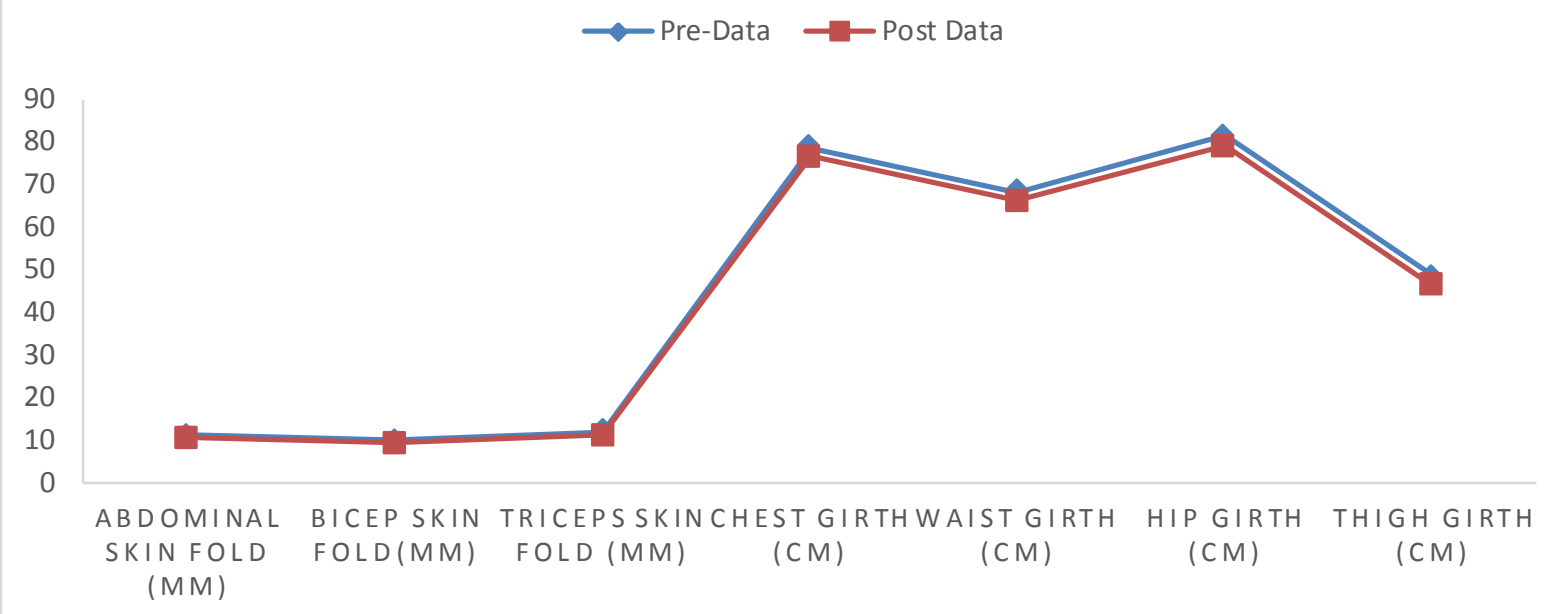

Significant at 0.5 levels.

Figure 03 shows significant difference in abdominal skin fold as pre-training and post-training $t=$ 2.03, sig $=0.01$, significant difference in bicep skin fold as pre-training and post-training $t=2.11, \mathrm{sig}$ $=0.01$, significant difference in chest girth $t=1.24, \mathrm{sig}=0.04$, and significant difference in hip girth $t$ $=1.18$, sig $=0.05$.

\section{Findings of the Study}

The results indicated that strength training was significantly affected the physical fitness and body composition of college girls. The considerable differences in the time of $30 \mathrm{~m}$ race, considerable improvement in agility, flexibility, and endurance have been found imperative worth of fitness training and exercise programs for college girls.

\section{Discussion and Conclusion}

There was a significant difference in reducing of body weight of college girls after strength training. Furthermore, physical fitness as agility, flexibility, endurance, standing broad jump, skipping has been significantly affected by strength training. Strength training reduces body fats and increases physical fitness, it may be concluded that after strength training, college girls become physically active along with a healthier lifestyle. The finding of this study was supported by the previous studies as the efficiency of exercises improves physical capability (Sonstroem \& Walker, 1973). Exercising such as aerobic activities, resistance training, walking, and few combinations are the ideal instances (Dishman et al., 1991).

It is concluded that strength training increases physical fitness and reduces the fat of college girls. The considerable differences in the period of $30 \mathrm{~m}$ race, in agility, flexibility, and endurance. There is evidence among youth that physical activities have declined in the last few decades (Kim et al., 2003). According to Australian research among 10 to 11 years students by comparing their physical activities also confirmed that the decrease in physical activities causes serious health and fitness problems. For example, the relationship between physical activity and obesity with energy intake status differs across different studies. Few studies have testified an inverse relation between adiposity level and activity while few also have not found any relationship (Marsh \& Peart, 1988).

\section{Recommendations}

In light of the findings of the present study, the researcher recommended that college girls regularly participate in fitness training to avoid obesity. An in-depth exploration of what is the effect of strength training on physical fitness and obesity of college girls more work is needed to fill the research gap in Pakistan.

\section{References}

Calestine, J., Bopp, M., Bopp, C. M., \& Papalia, Z. (2017). College student work habits are related to physical activity and fitness. International Journal of Exercise Science, 10(7), 1009.

Dishman, R. K. (1991). Increasing and maintaining exercise and physical activity. Behaviour Therapy, 22(3), 345-378.

Downs, A., \& Ashton, J. (2011). Vigorous physical activity, sports participation, and athletic identity: 
Implications for mental and physical health in college students. Journal of Sport Behavior, 34(3), 228.

Egli, T., Bland, H. W., Melton, B. F., \& Czech, D. R. (2011). Influence of age, sex, and race on college students' exercise motivation of physical activity. Journal of American College Health, 59(5), 399-406.

Farren, G. L., Zhang, T., Martin, S. B., \& Thomas, K. T. (2017). Factors related to meeting physical activity guidelines inactive college students: A social cognitive perspective. Journal of American College Health, 65(1), 10-21.

French, S. A., \& Jeffery, R. W. (1994). Consequences of dieting to lose weight: effects on physical and mental health. Health Psychology, 13(3), 195.

Fu, Y., Gao, Z., Hannon, J., Shultz, B., Newton, M., \& Sibthorp, J. (2013). Influence of a health-related physical fitness model on students' physical activity, perceived competence, and enjoyment. Perceptual and Motor Skills, 117(3), 956-970.

Gutin, B., Cucuzzo, N., Islam, S., Smith, C., \& Stachura, M. E. (1996). Physical training, lifestyle education, and coronary risk factors in obese girls. Medicine \& Science in Sports \& , 28(1), 19-23.

Haq, M. Z. U., Iqbal, A., Afzal, A., Ahmad, H., Abbas, S., \& Yaqoob, M. (2019). Anthropometric Characteristics and Physical Fitness of Urban and Rural 8-10 Years Old School Girls of Bahaw alpur, Pakistan. International Journal of Physiotherapy, 6(2), 46-51

Kim, H.-Y. P., Frongillo, E. A., Han, S.-S., Oh, S.-Y., Kim, W.-K., Jang, Y.-A., Won, H.-S., Lee, H.-S., Kim, S.-H., \& Han, S.-S. (2003). The academic performance of Korean children is associated with dietary behaviors and physical status. Asia Pacific Journal of Clinical Nutrition, 12(2).

Lauderdale, M. E., Yli-Piipari, S., Irwin, C. C., \& Layne, T. E. (2015). Gender differences regarding motivation for physical activity among college students: A self-determination approach. The Physical Educator, 72(5).

Lockwood, P., \& Wohl, R. (2012). The impact of a 15-week lifetime wellness course on behavior change and self-efficacy in college students. College Student Journal, 46(3), 628-641.

Marsh, H. W., \& Peart, N. D. (1988). Competitive and cooperative physical fitness training programs for girls: Effects on physical fitness and multidimensional self-concepts. Journal of Sport and Exercise Psychology, 10(4), 390-407.

Meckel, Y., Galily, Y., Nemet, D., \& Eliakim, A. (2011). Changes in weight indexes and aerobic fitness of physical education students over three years of college. Journal of Human Sport and Exercise, 6(1), 112-121.

Pope, L., \& Harvey-Berino, J. (2013). Burn and earn: A randomized controlled trial incentivizing exercise during the fall semester for college first-year students. Preventive Medicine, 56(3-4), 197-201.

Puhl, R. M., \& Heuer, C. A. (2009). The stigma of obesity: a review and update. Obesity, 17(5), 941-964.

Sanchez-Vaznaugh, E. V, Sánchez, B. N., Rosas, L. G., Baek, J., \& Egerter, S. (2012). Physical education policy compliance and children's physical fitness. American Journal of Preventive Medicine, 42(5), 452-459.

Sibley, B. A., Hancock, L., \& Bergman, S. M. (2013). Univ ersity students' exerc ise behavioral regulation, motives, and physical fitness. Perceptual and Motor Skills, 116(1), 322-339.

Sonstroem, R. J., \& Walker, M. I. (1973). Relationship of attitudes and locus of control to exercise and physical fitness. Perceptual and Motor Skills, 36(3_suppl), 1031-1034.

Steiber, N. (2016). Strong or weak handgrip? Normative reference values for the German population across the life course stratified by sex, age, and body height. PloS one, 11(10), e0163917.

Stice, E., Shaw, H., \& Marti, C. N. (2006). A meta-analytic review of obesity prevention programs for children and adolescents: the skinny on interventions that work. Psychological Bulletin, 132(5), 667.

Weinstock, J., Capizzi, J., Weber, S. M., Pescatello, L. S., \& Petry, N. M. (2014). Exercise as an intervention for sedentary hazardous drinking college students: A pilot study. Mental Health and Physical Activity, 7(1), 55-62.

Zhao, W., Wei, Y., \& Li, S. (2011). Comparative study on physical fitness of male college athletes for a different specific event. Journal of Harbin Institute of Physical Education, 3.

Zheng, G., Li, M., Lan, X., Yan, X., Lin, Q., Chen, L., Tao, J., Zheng, X., Li, J., \& Chen, B. (2013). The effect of Baduanjin exercise on physical and psychological wellbeing of college students: study protocol for a randomized controlled trial. Trials, 14(1), 422. 